\title{
Implementation of Isogeometric Fast Multipole Boundary Element Methods for 2D Half-Space Acoustic Scattering Problems with Absorbing Boundary Condition
}

\author{
Leilei Chen \\ College of Architecture and Civil Engineering \\ Xinyang Normal University, Xinyang 464000, Henan, P. R. China \\ chenllei@mail.ustc.edu.cn \\ Steffen Marburg \\ Institute of Vibroacoustics of Vehicles and Machines \\ Faculty of Mechanical Engineering, Technical University of Munich \\ Boltzmannstr. 15, 85748 Garching bei München, Germany \\ Wenchang Zhao, Cheng Liu and Haibo Chen \\ Department of Modern Mechanics \\ University of Science and Technology of China \\ CAS Key Laboratory of Mechanical Behavior and Design of Materials \\ Hefei 230026, Anhui, P. R. China
}

Received 12 October 2017

Accepted 19 February 2018

Published 12 July 2018

\begin{abstract}
Isogeometric Analysis (IGA), which tries to bridge the gap between Computer Aided Engineering (CAE) and Computer Aided Design (CAD), has been widely proposed in recent research. According to the concept of IGA, this work develops a boundary element method (BEM) using non-Uniform Rational B-Splines (NURBS) as basis functions for the 2D half-space acoustic problems with absorbing boundary condition. Fast multipole method (FMM) is applied to accelerate the solution of an isogeometric BEM (IGA-BEM). Several examples are tested and it is shown that this advancement on isogeometric fast multipole boundary element method improves the accuracy of simulations.
\end{abstract}

Keywords: Isogeometric analysis; boundary element method; fast multipole method; half-space acoustics.

\section{Introduction}

The BEM is a developing computational method for engineering and a commonly used numerical method in different engineering fields, including fluid mechanics, acoustics, electromagnetics and fracture mechanics. ${ }^{112}$ As an alternative method to the finite element

This is an Open Access article published by World Scientific Publishing Company. It is distributed under the terms of the Creative Commons Attribution 4.0 (CC-BY) License. Further distribution of this work is permitted, provided the original work is properly cited. 


\section{Chen et al.}

method (FEM) and the finite difference method (FDM), the BEM has several advantages. The most distinct feature is that it can reduce the two-dimensional problem down to a onedimensional boundary and a three-dimensional problem down to a two-dimensional surface. Another advantage is that the BEM could gain the same level of accuracy with less number of elements compared with other methods when the fast multipole method (FMM) is applied to accelerate the solution of matrix-vector! 3

The BEM has been widely used to acoustic problems because it provides an excellent accuracy and easy mesh generation. 10 12 In particular, for exterior acoustic problems, the Sommerfeld radiation condition at infinity is automatically satisfied. Actually, for the BEM, the Helmholtz governing partial differential equation is first converted into an integral equation which can then be discretized through an appropriate basis and a suitable discretization method. However, for the exterior acoustic problem, the use of conventional single boundary integral equation can produce nonuniqueness problem. The Burton-Miller method, which is obtained by combining the conventional boundary integral equation and its normal derivative equation, can be used successfully to overcome this difficulty!13 15

Conventional numerical methods, such as the FEM and BEM, use Lagrangian polynomials to approximate the geometry of the boundary, and, thus, may produce an additional error. An alternative method is applying spline curve used in computer aided design (CAD) software to represent the boundary shape, and applying the functions used to describe the geometry in CAD software to approximate the unknown physics fields in numerical simulations. This concept is called IGA 16

Recent work on the IGA-BEM has focused on improving the numerical computing accuracy by using spline curve to represent the boundary shape. In Ref. 16, the concept of isogeometric analysis (IGA) is proposed in detail and then applied into the FEM. The result shows that the basis functions are complete with respect to affine transformations, and it means that all rigid body motions and constant strain states can be exactly represented.16 Several early efforts applied B-spline to the BEM to solve Laplace problems. ${ }^{[2]}[23$ A spline is a piecewise polynomial function and a spline with knots vector will represent parametric curves with guaranteed continuity. A knot vector is a set of coordinates in the parametric space. A B-spline is formed by a number of curve segments which can maintain continuity. However, it is difficult for a low order B-spline to represent accurately circle, ellipse and other complex structures. Using NonUniform Rational B-Splines (NURBS), designers can obtain more control of the represented curve without increasing the number of control points or increasing the curve degree. Several researchers 24 applied the BEM and NURBS to 2D elastostatic analysis, and Peng et al. $\stackrel{27}{27}$ applied the BEM and NURBS to 3D static fracture

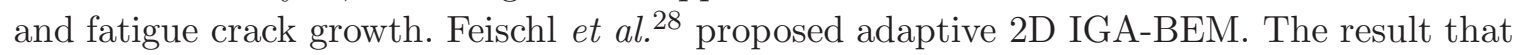
the proposed adaptive strategy leads to optimal convergence can also be found in Ref. 28. Several researchers ${ }^{29}$ applied the IGA-BEM to 2D acoustic problems, and then extended this method into 3D acoustic problems. 34 Optimization algorithm based on IGA has been widely applied to the optimization analysis for complex practical problems 455 The previous research shows the high application potential and validity of the IGA-BEM for practical problems. 
This research extends the IGA-BEM into the 2D half-space acoustic problems with absorbing boundary condition. In order to improve the computing efficiency and decrease the storage, isogeometric fast multipole boundary element method (IGA-FMBEM) obtained by combining the IGA-BEM and FMM is applied for the 2D half-space acoustic problems in this paper. Several examples are tested and it is shown that this advancement on the IGA-FMBEM improves the accuracy of simulations.

\section{Representation of Boundary}

It is the key idea of IGA to bring together the fields of design and analysis into a unified framework by using parametric functions that are predominant in CAD. We focus on describing NURBS functions which are used to represent geometries in CAD. B-splines and NURBS can both describe the curves (or surfaces), and they are both parametric.

A single closed curve with B-spline basis functions of degree $p$ is generated to represent the boundary, where the parameter $\xi \in[0,1]$ along the curve to be generated is introduced. A set of knots on the curve are given to generate a knot vector $\Xi=\left[\xi_{0}, \xi_{1}, \ldots, \xi_{m}\right]$, which is a nondecreasing sequence of real numbers given in parameter space, where $\xi_{i} \in \mathbb{R}$ is the $i$ th knot, $m$ is the length of the knot vector, $m=n+p+1$, and $n$ is the number of basic functions. Turning attention toward B-spline and NURBS basis functions, the coordinate at any point on the curve constructed can be expressed by

$$
\mathbf{x}(\xi)=\sum_{i=0}^{n} R_{i, p}(\xi) \mathbf{P}_{i},
$$

where vector $\mathbf{P}_{i}$ denotes the coordinate at the control points, $R_{i, p}(\xi)$ are NURBS basis functions, defined as

$$
R_{i, p}(\xi)=\frac{N_{i, p}(\xi) w_{i}}{W(\xi)}
$$

with

$$
W(\xi)=\sum_{i=0}^{n} w_{i} N_{i, p}(\xi)
$$

where $w_{i}$ denotes a weight associated with the control point $\mathbf{P}_{i}$. B-spline basis functions $N_{i, p}(\xi)$ can be expressed as

$$
N_{i, 0}(\xi)= \begin{cases}1 & \text { if } \xi_{i} \leq \xi<\xi_{i+1} \\ 0 & \text { otherwise }\end{cases}
$$

then, for $p=1,2,3, \ldots$

$$
N_{i, p}(\xi)=\frac{\xi-\xi_{i}}{\xi_{i+p}-\xi_{i}} N_{i, p-1}(\xi)+\frac{\xi_{i+p+1}-\xi}{\xi_{i+p+1}-\xi_{i+1}} N_{i+1, p-1}(\xi) .
$$

Actually, it is hard for B-spline with lower order to model complex objects. For example, it is very difficult to model accurately circles and ellipses using low order B-spline. If we wish 


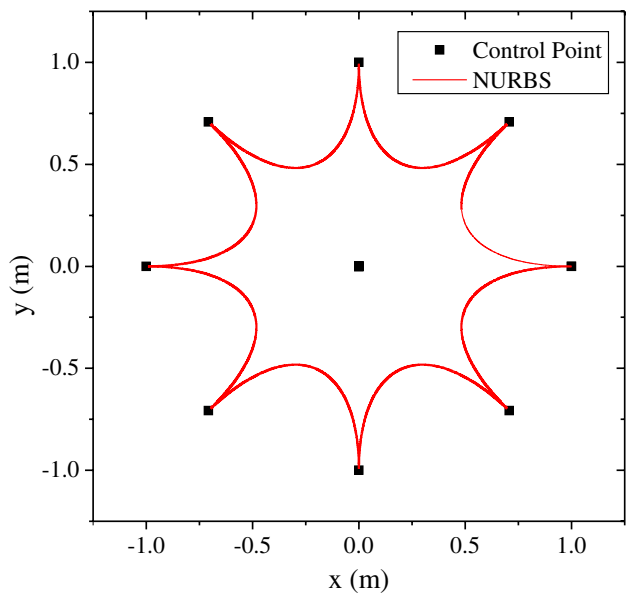

(a) Octagonal column

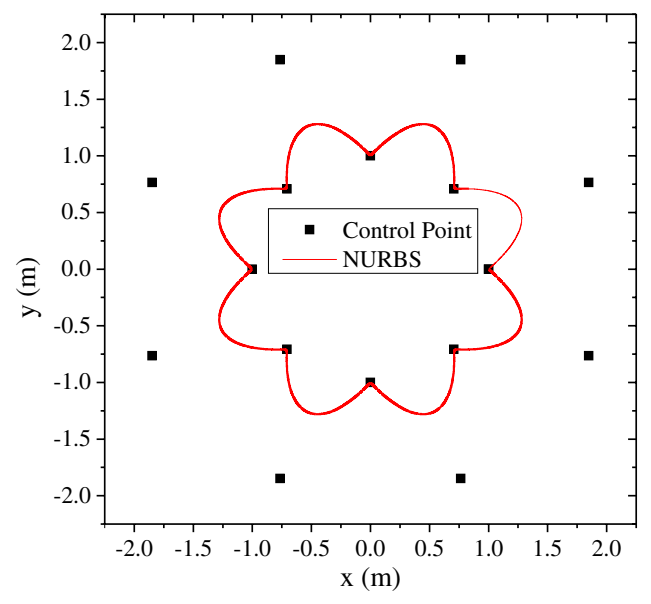

(b) Eight blade column

Fig. 1. Complex structures represented by NURBS curve of degree 2 generated by 17 control points; the knot vector $\Xi=\{0,0,0,1,1,2,2,3,3,4,4,5,5,6,6,7,7,8,8,8\} / 8$.

to shift the curve closer or farther to the control point without introducing new control points and changing the position of the existing control point and, of course, not losing continuity. A good method to achieve the aim is to use rational B-splines that are also called NURBS. Rational B-spline means the objects modeled by the spline can be expressed by rational polynomials. The solution is to introduce homogeneous coordinates into the function of spline. It associates a weight with each control point and we can change the curve by changing the weight of the corresponding control point.

In Fig. 1 two complex structures, which are called octagonal column and eight blade column respectively, are constructed to show the flexibility and applicability of NURBS curves. Herein, the data about the control points and the associated weights are listed in Table 1.

\section{Conventional BEM}

We propose a collocational boundary element method using linear and quadratic Lagrangian functions for the Helmholtz equation in 2D. To make things simple, we focus on the acoustic scattering problem from noise barrier with absorbing material. The boundary integral equation (BIE) and normal derivative equation (NDBIE) based on Helmholtz equation for half-space problem can be expressed as, respectively

$$
c(r) p\left(r, r_{0}\right)=p_{i}+\int_{S}\left[\frac{\partial G\left(r_{s}, r\right)}{\partial n\left(r_{s}\right)}-i k \beta\left(r_{s}\right) G\left(r_{s}, r\right)\right] p\left(r_{s}, r_{0}\right) d s\left(r_{s}\right),
$$

and

$$
c(r) q\left(r, r_{0}\right)=\frac{\partial p_{i}}{\partial n(r)}+\int_{S}\left[\frac{\partial^{2} G\left(r_{s}, r\right)}{\partial n\left(r_{s}\right) n(r)}-i k \beta\left(r_{s}\right) \frac{\partial G\left(r_{s}, r\right)}{\partial n(r)}\right] p\left(r_{s}, r_{0}\right) d s\left(r_{s}\right)
$$


Table 1. Data about control point and the associated weight for eight blade column and octagonal column.

\begin{tabular}{crrrrrrr}
\hline & \multicolumn{3}{c}{ Eight Blade Column } & & \multicolumn{3}{c}{ Octagonal Column } \\
\cline { 2 - 3 } Control Point & \multicolumn{1}{c}{$x$} & \multicolumn{1}{c}{$y$} & Weight $w$ & & \multicolumn{1}{c}{$x$} & \multicolumn{1}{c}{$y$} & Weight $w$ \\
\hline $\mathrm{P}_{0}$ & 1.000 & 0.000 & 1.000 & & 1.000 & 0.000 & 1.000 \\
$\mathrm{P}_{1}$ & 1.848 & 0.765 & 0.707 & & 0.000 & 0.000 & 0.707 \\
$\mathrm{P}_{2}$ & 0.707 & 0.707 & 1.000 & & 0.707 & 0.707 & 1.000 \\
$\mathrm{P}_{3}$ & 0.765 & 1.848 & 0.707 & & 0.000 & 0.000 & 0.707 \\
$\mathrm{P}_{4}$ & 0.000 & 1.000 & 1.000 & & 0.000 & 1.000 & 1.000 \\
$\mathrm{P}_{5}$ & -0.765 & 1.848 & 0.707 & & 0.000 & 0.000 & 0.707 \\
$\mathrm{P}_{6}$ & -0.707 & 0.707 & 1.000 & & -0.707 & 0.707 & 1.000 \\
$\mathrm{P}_{7}$ & -1.848 & 0.765 & 0.707 & & 0.000 & 0.000 & 0.707 \\
$\mathrm{P}_{8}$ & -1.000 & 0.000 & 1.000 & & -1.000 & 0.000 & 1.000 \\
$\mathrm{P}_{9}$ & -1.848 & -0.765 & 0.707 & & 0.000 & 0.000 & 0.707 \\
$\mathrm{P}_{10}$ & -0.707 & -0.707 & 1.000 & & -0.707 & -0.707 & 1.000 \\
$\mathrm{P}_{11}$ & -0.765 & -1.848 & 0.707 & & 0.000 & 0.000 & 0.707 \\
$\mathrm{P}_{12}$ & 0.000 & -1.000 & 1.000 & & 0.000 & -1.000 & 1.000 \\
$\mathrm{P}_{13}$ & 0.765 & -1.848 & 0.707 & & 0.000 & 0.000 & 0.707 \\
$\mathrm{P}_{14}$ & 0.707 & -0.707 & 1.000 & & 0.707 & -0.707 & 1.000 \\
$\mathrm{P}_{15}$ & 1.848 & -0.765 & 0.707 & & 0.000 & 0.000 & 0.707 \\
$\mathrm{P}_{16}$ & 1.000 & 0.000 & 1.000 & 1.000 & 0.000 & 1.000 \\
\hline
\end{tabular}

where the coefficient $c(r)$ is determined by the boundary geometry at the source point $r, r_{s}$ denotes the field point on the boundary, $r_{0}=\left(x_{0}, y_{0}\right)$ is the acoustic source point outside the boundary, $\beta$ is the acoustic admittance, $p_{i}$ is the incident acoustic pressure. The Green function $G\left(r, r_{0}\right)$ can be expressed as

$$
G\left(r, r_{0}\right)=\frac{i}{4}\left[H_{0}^{(1)}\left(k\left|r_{0}-r\right|\right)+H_{0}^{(1)}\left(k\left|\overline{r_{0}}-r\right|\right)\right],
$$

where $\overline{r_{0}}=\left(x_{0},-y_{0}\right)$ is the mirror point of $r_{0}$. In the conventional BEM, $p\left(r_{s}, r_{0}\right)$ in every discretized boundary element can be obtained by using Lagrangian interpolation function.

$$
p\left(r_{s}, r_{0}\right)=\sum_{k=1}^{m} \Phi_{k} p\left(r_{k}, r_{0}\right)
$$

where $m$ denotes the number of interpolation nodes in every boundary element, $\Phi$ denotes the Lagrangian interpolation function, $r_{k}$ denotes the $k$ th nodal point. The Lagrangian interpolation function $\Phi$ for constant, linear, and quadratic boundary element are given, respectively $\underline{10143}$

(1) For constant element

$$
m=1 \quad \text { and } \quad \Phi=1
$$

(2) For linear element

$$
\Phi_{1}=\frac{1}{2}\left(1-\frac{\xi}{\alpha}\right), \quad \Phi_{2}=\frac{1}{2}\left(1+\frac{\xi}{\alpha}\right)
$$


L. Chen et al.

where $\xi$ means the local coordinate of the field point $r_{s}$, and $\alpha$ denotes the position of interpolation nodes on the discontinuous element. When $\alpha=1$, Eq. (10) denotes expression of the interpolation functions for linear continuous element.

(3) For quadratic element.

$$
\Phi_{1}=\frac{\xi}{2 \alpha}\left(\frac{\xi}{\alpha}-1\right), \quad \Phi_{2}=1-\frac{\xi^{2}}{\alpha^{2}}, \quad \Phi_{3}=\frac{\xi}{2 \alpha}\left(\frac{\xi}{\alpha}+1\right)
$$

Similar as linear element, Eq. (11) denotes expression of the interpolation functions for quadratic continuous element when $\alpha=1$.

Figure 2 presents distribution of nodal points and geometrical nodes for a set of conventional boundary element based on Lagrangian interpolation function. The interpolation shape functions for different types of boundary elements are shown in Figs. 3 and 4.
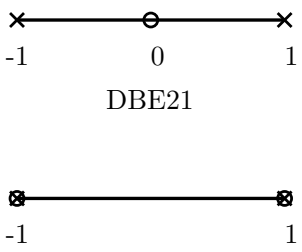

CBE22
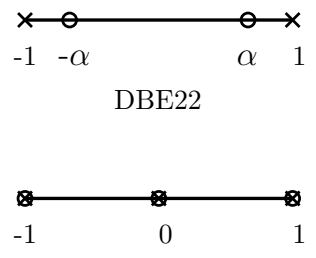

CBE33

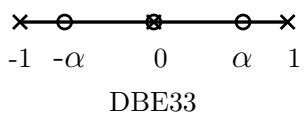

$\times \quad$ geometrical node

- interpolation node

Fig. 2. Distribution of nodal points in any boundary element based on Lagrangian interpolation function. For discontinuous boundary elements, interpolation nodes are located inside the elements and the expressions of the interpolation functions are dependent on the position of the node inside the elements. The optional nodal position is found at the zeroes orthogonal polynomials $\underline{43 \mid 44}$

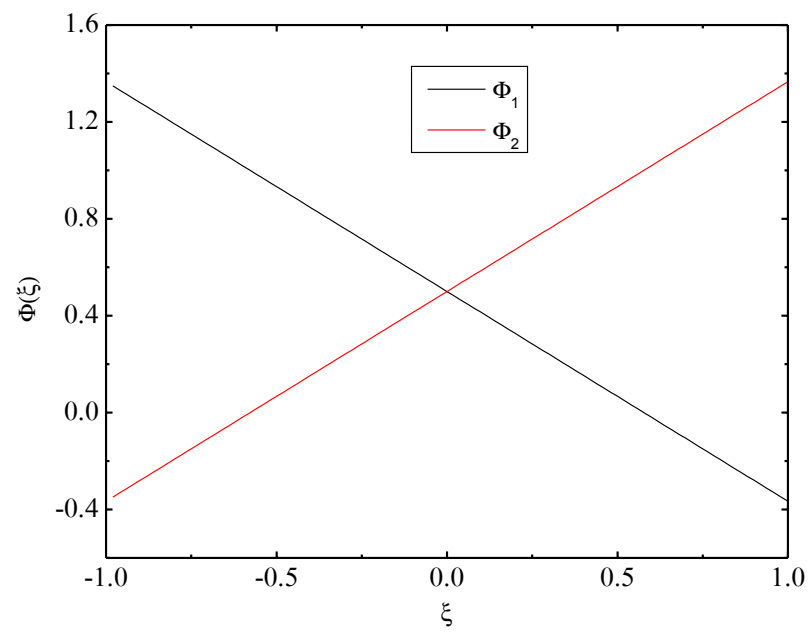

Fig. 3. Lagrangian interpolation function for discontinuous linear boundary element. The parameter of optimal nodal position is set as 0.5773 . 


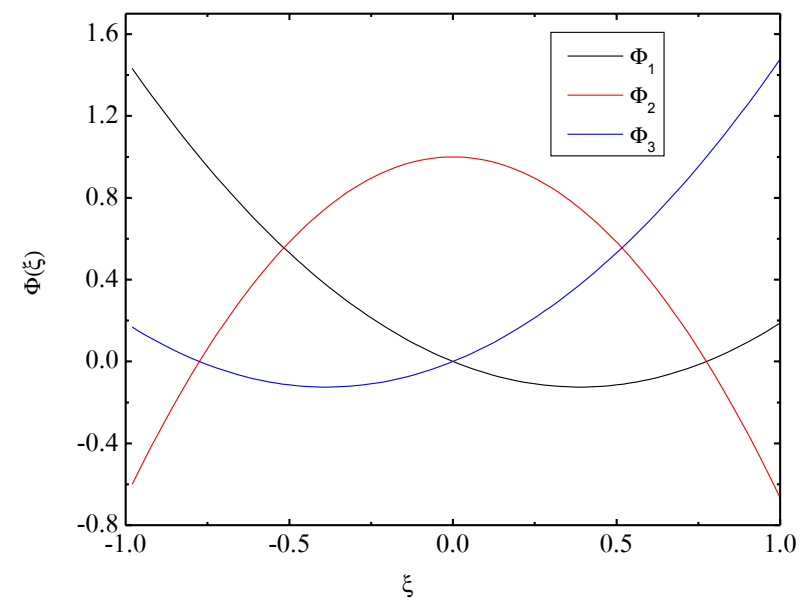

Fig. 4. Lagrangian interpolation function for discontinuous quadratic boundary element. The parameter of optimal nodal position is set as 0.7746 .

\section{FMBEM for Half-Space Acoustic Problem with Absorbing Material}

It is well-known that the conventional BEM generates a full coefficient matrix, which takes $N^{2}$ calculation operation for a problem with $N$ degree of freedom. However, the use of the FMM avoids the direct solution of the coefficient matrix, and reduces the computing operation from $N^{2}$ to $N$ or $N \log N$, and so decreases significantly the required time of numerical solution. Actually, three different procedures of the FMM can be found according to the difference in the form of expansion of the Green's function, see Refs. 45, 46 and 47. For the full-space problem, only the tree structure containing the whole sound barrier structure is produced for the FMM operation. However, for the half-space problem, due to the existence of the mirrored domain, an extra tree structure needs to be produced in order to implement the FMM operation used for the solution of the boundary integral in mirrored domain. The boundary integrals in the two domains are solved separately. This method can be used successfully for the solution of the half-space problem, but it brings extra computing operation and increases the computational complexity. An alternative of this method is to create a mirrored source point, but not a mirrored integral domain. That means the calculation for the original boundary integral in the mirrored domain may be turned into the calculation for the boundary integral with a mirrored source point in the real structure domain.

Implementation process of the FMM is introduced in brief. In a first step, the tree structure is generated. A square containing the boundary $S$ is introduced after discretization of the boundary. This square is divided into four equally sized child squares. Keep dividing a square in this way until the number of elements in that square is less than a specified number. The second step is called upward pass to calculate the multipole moments of each square. For this, the Green function is expanded as follows ${ }^{778}$.

$$
G\left(r_{s}, r\right)=-\frac{i}{4}\left[\sum_{n=-\infty}^{\infty} O_{n}\left(\overrightarrow{r_{s}^{1} r}\right) I_{-n}\left(\overrightarrow{r_{s}^{1} r_{s}}\right)+\sum_{n=-\infty}^{\infty} O_{n}\left(\overrightarrow{r_{s}^{1} \bar{r}}\right) I_{-n}\left(\overrightarrow{r_{s}^{1} r_{s}}\right)\right],
$$




\section{Chen et al.}

where $r_{s}^{1}$ is very close to $r_{s}$ and $\left|r_{s}^{1} r\right| \gg\left|r_{s}^{1} r_{s}\right|$. The functions $O_{n}$ and $I_{n}$ are expressed as

$$
O_{n}\left(\overrightarrow{r_{s}^{1} r}\right)=i^{n} H_{n}^{(1)}\left(k R_{1}\right) e^{i n \theta_{1}}
$$

and

$$
I_{n}\left(\overrightarrow{r_{s}^{1} r_{s}}\right)=(-i)^{n} J_{n}^{(1)}\left(k R_{2}\right) e^{i n \theta_{2}},
$$

where $J_{n}$ is the $n$th Bessel function, and $(R, \theta)$ denotes the polar coordinates of a vector. When $r$ is far away from $S_{0}$ which is a subset of the boundary $S$, the boundary integral in Eqs. (6) and (7) can be rewritten as

$$
A_{1}=\int_{S_{0}}\left[\frac{\partial G\left(r_{s}, r\right)}{\partial n\left(r_{s}\right)}-i k \beta\left(r_{s}\right) G\left(r_{s}, r\right)\right] p\left(r_{s}, r_{0}\right) d s\left(r_{s}\right),
$$

and

$$
B_{1}=\int_{S_{0}}\left[\frac{\partial^{2} G\left(r_{s}, r\right)}{\partial n\left(r_{s}\right) n(r)}-i k \beta\left(r_{s}\right) \frac{\partial G\left(r_{s}, r\right)}{\partial n(r)}\right] p\left(r_{s}, r_{0}\right) d s\left(r_{s}\right) .
$$

Substituting Eq. (12) into Eqs. (15) and (16), we obtain the following two equations:

$$
\begin{aligned}
& A_{1}=-\frac{i}{4}\left[\sum_{n=-\infty}^{\infty} O_{n}\left(\overrightarrow{r_{s}^{1} r}\right)+O_{n}\left(\overrightarrow{r_{s}^{1} \bar{r}}\right)\right] M_{n}\left(r_{s}^{1}\right), \\
& B_{1}=-\frac{i}{4}\left[\sum_{n=-\infty}^{\infty} \frac{\partial O_{n}\left(\overrightarrow{r_{s}^{1} r}\right)}{\partial n(r)}+\frac{\partial O_{n}\left(\overrightarrow{r_{s}^{1} \bar{r}}\right)}{\partial n(r)}\right] M_{n}\left(r_{s}^{1}\right),
\end{aligned}
$$

where $M_{n}$ denotes the coefficient of the multipole expansion and is expressed as

$$
M_{n}\left(r_{s}^{1}\right)=\int_{S_{0}}\left[\frac{\partial I_{-n}\left(\overrightarrow{r_{s}^{1} r_{s}}\right)}{\partial n\left(r_{s}\right)}-i k \beta I_{-n}\left(\overrightarrow{r_{s}^{1} r_{s}}\right)\right] p\left(r_{s}, r_{0}\right) d s\left(r_{s}\right)
$$

Then, the multipole moment of the higher level square is obtained by using the transfer operation of the multipole moment of the lower level square, that is called the M2M, as follows

$$
M_{n}\left(r_{s}^{2}\right)=\sum_{m=-\infty}^{+\infty} I_{-n+m}\left(\overrightarrow{r_{s}^{2} r_{s}^{1}}\right) M_{m}\left(r_{s}^{1}\right)
$$

After obtaining the multipole moments of all divided squares, we need to implement the downward pass operation. The whole boundary integral is divided into two parts. One is called the nearfield integral that is solved by using conventional boundary integral methods. 
The other one is called the far-field integral that is solved by using the M2L and L2L transfer operation, as follows

$$
L_{n}\left(r^{1}\right)=\sum_{m=-\infty}^{+\infty}(-1)^{n} O_{n-m}\left(\overrightarrow{r_{s}^{2} r^{1}}\right) M_{-m}\left(r_{s}^{2}\right),
$$

and

$$
L_{n}\left(r^{2}\right)=\sum_{m=-\infty}^{+\infty} I_{n-m}\left(\overrightarrow{r^{2} r^{1}}\right) L_{m}\left(r^{1}\right),
$$

where $r_{s}^{2}$ is located close to $S_{0}, r^{1}$ and $r^{2}$ close to $r$.

Finally, we can obtain the following new expressions for the boundary integrals

$$
\begin{aligned}
& A_{1}=\sum_{n=-\infty}^{\infty}\left[I_{-n}\left(\overrightarrow{r^{2} r}\right) L_{n}\left(r^{2}\right)+I_{-n}\left(\overrightarrow{r^{3} \bar{r}}\right) L_{n}\left(r^{3}\right)\right] \\
& B_{1}=\sum_{n=-\infty}^{\infty}\left[\frac{\partial I_{-n}\left(\overrightarrow{r^{2} r}\right)}{\partial n(r)} L_{n}\left(r^{2}\right)+\frac{\partial I_{-n}\left(\overrightarrow{r^{3}} \bar{r}\right)}{\partial n(r)} L_{n}\left(r^{3}\right)\right],
\end{aligned}
$$

where $r^{3}$ is close to $\bar{r}$. For more details on this, the reader is referred to Refs. 7 and 8 .

\section{Isogeometric FMBEM}

In the implementation process of the IGA-BEM, the sound pressure at the boundary is interpolated by using the NURBS basis functions. Although a few geometric control points can generate very accurately a simple curve by using NURBS basis functions, large errors will be produced when the same number of control points is used for the approximation of field variables. In order to overcome this problem, h-refinement operations via knot insertion without changing the geometry are implemented. The insertion of a new knot $\bar{\xi} \in\left[\xi_{k}, \xi_{k+1}\right]$ leads to a modification of control points as

$$
\bar{P}_{i}=\alpha_{i} P_{i}+\left(1-\alpha_{i}\right) P_{i-1}
$$

with

$$
\alpha_{i}= \begin{cases}1, & \leq i \leq k-p, \\ \frac{\bar{\xi}-\xi_{i}}{\xi_{i+p}-\xi_{i}}, & k-p+1 \leq i \leq k, \\ 0, & k+1 \leq i .\end{cases}
$$

where $\overline{\mathbf{P}}$ stands for the added control points. The existing knot values may be repeated in this algorithm, and the continuity of the basis will be decreased.16

After that, we obtain a new knot vector $\Xi_{f}$ and a set of particular control points which are used to approximate the field variable, where $n_{f}$ denotes the number of the new control 


\section{Chen et al.}

points and is equal to the system's degree of freedom after discretization. By using NURBS basis functions for interpolation, we obtain the sound pressure around the boundary as follows:

$$
p(\xi)=\sum_{i=0}^{n_{f}} R_{i, p_{f}}(\xi) P_{i}
$$

where $p_{i}$ denotes the sound pressure at the new particular control point which may or may not lie on the boundary, and $p_{f}$ is the order.

In the conventional BEM, the normal practice of collocation at nodal positions is valid. However, this way is no longer valid in the IGA-BEM because the control points may not lie on the boundary. To overcome this difficulty, we use the Greville abscissae definition to define the position of collocation points in parameter space, as follows:

$$
\bar{\xi}_{j}=\frac{\xi_{j+1}+\xi_{j+2}+\cdots+\xi_{j+p_{f}}}{p_{f}}, \quad j=0,1, \ldots, n_{f} .
$$

Using Eq. (27), we obtain the pressure at the collocation points. By substituting Eq. (27) into Eqs. (66) and (7), we obtain the following boundary integral equation for IGA-BEM

$$
\begin{aligned}
c\left(r\left(\bar{\xi}_{i}\right)\right) \sum_{j=0}^{n_{f}} R_{j, p_{f}}\left(\bar{\xi}_{i}\right) P_{j}= & G\left(r\left(\bar{\xi}_{i}\right), r_{0}\right)+\sum_{e=1}^{N_{e}} \sum_{j=0}^{n_{f}}\left\{\int _ { \xi _ { e } } ^ { \xi _ { e + 1 } } \left[\frac{\partial G\left(r_{s}(\xi), r\left(\bar{\xi}_{i}\right)\right)}{\partial n\left(r_{s}(\xi)\right)}\right.\right. \\
& \left.\left.-i k \beta\left(r_{s}(\xi)\right) G\left(r_{s}(\xi), r\left(\bar{\xi}_{i}\right)\right)\right] R_{j, p_{f}}(\xi) J(\xi) d \xi\right\} p_{j}
\end{aligned}
$$

and

$$
\begin{aligned}
i k \beta c\left(r\left(\bar{\xi}_{i}\right)\right) \sum_{j=0}^{n_{f}} R_{j, p_{f}}\left(\bar{\xi}_{i}\right) P_{j}= & \frac{\partial G\left(r\left(\bar{\xi}_{i}\right), r_{0}\right)}{\partial n\left(r\left(\bar{\xi}_{i}\right)\right)}+\sum_{e=1}^{N_{e}} \sum_{j=0}^{n_{f}}\left\{\int _ { \xi _ { e } } ^ { \xi _ { e + 1 } } \left[\frac{\partial^{2} G\left(r_{s}(\xi), r\left(\bar{\xi}_{i}\right)\right)}{\partial n\left(r_{s}(\xi)\right) n\left(r\left(\bar{\xi}_{i}\right)\right)}\right.\right. \\
& \left.\left.-i k \beta\left(r_{s}(\xi)\right) \frac{\partial G\left(r_{s}(\xi), r\left(\bar{\xi}_{i}\right)\right)}{\partial n\left(r\left(\bar{\xi}_{i}\right)\right)}\right] R_{j, p_{f}}(\xi) J(\xi) d \xi\right\} p_{j}
\end{aligned}
$$

where $\frac{\partial p}{\partial n}=i k \beta p, N_{e}$ is the number of NURBS element discretizing the boundary curve; $\left[\xi_{e}, \xi_{e+1}\right]$ denotes a NURBS element and is an interval between two consequent nonrepeating knots in parameter space; $J(\xi)$ stands for the Jacobian. Singular integrals exist in Eqs. (29) and (30) when $\bar{\xi}_{i} \in\left[\xi_{e}, \xi_{e+1}\right]$, and special treatment needs to be done to eliminate the singular integrals, see Appendix A.

With the FMM applied to accelerate the solution of IGA-BEM, Eq. (19) is rewritten as

$$
M_{n}\left(r_{s}^{1}\right)=\sum_{e=1}^{N_{0}} \sum_{j=0}^{n_{f}} \int_{\xi_{e}}^{\xi_{e+1}}\left[\frac{\partial I_{-n}\left(\overrightarrow{r_{s}^{1} r_{s}}\right)}{\partial n\left(r_{s}\right)}-i k \beta I_{-n}\left(\overrightarrow{r_{s}^{1} r_{s}}\right)\right] R_{j, p_{f}(\xi)} p_{j} J(\xi) d \xi
$$

where the subset $S_{0}$ contains $N_{0}$ NURBS elements. Similar to the implementation process of the conventional FMBEM, the IGA-FMBEM also needs operation of the M2M, M2L, and 
L2L. Finally, using Eqs. (23) and (24), we can obtain the solution of the far-field boundary integral based on the NURBS basis functions.

\section{Numerical Examples}

In this example, we consider the acoustic scattering of a plane incident wave with a unit amplitude on a rigid cylindrical shell with a radius $a=1 \mathrm{~m}$ centered at point $(0,0)$. The medium in the domain is air with density $\rho=1.2 \mathrm{~kg} / \mathrm{m}^{3}$. The wave speed is $340 \mathrm{~m} / \mathrm{sec}$. The incident wave is traveling along positive $\mathbf{x}$-axis. By comparing the numerical solution with an analytical solution, we can demonstrate the validity and accuracy of the algorithm proposed in this paper. This model can be simplified to be $2 \mathrm{D}$ acoustic problem with a circle boundary. The analytical solution of the acoustic pressure at any point located at $(r, \theta)$ is given as

$$
\phi(r, \theta)=-\sum_{n=0}^{\infty} \varepsilon_{n} i^{n} \frac{J_{n}^{\prime}(k a)}{H_{n}^{(1) \prime}(k a)} H_{n}^{(1)}(k r) \cos (n \theta),
$$

where $\varepsilon_{n}$ denotes the Neumann symbols, i.e. $\varepsilon_{0}=1 ; \varepsilon_{n}=2$ when $n$ is greater than 0 . ( $)^{\prime}$ stands for the differentiation with respect to $k a$.

Figure 5] shows a comparison between analytical sound pressure and numerical solution based on the IGA-FMBEM, where the test points are located uniformly on a circle with a radius $r=4$. Figures 5(a) and 5(b) show the real and the imaginary parts of the sound pressure at test points, respectively. Figures [6 and 7 show the real and the imaginary parts of the sound pressure at a point $(4,0)$ with frequency, respectively. By observing these figures, we find that the numerical solution obtained using the IGA-FMBEM is in good agreement with the analytical solution, and it confirms the correctness and validity of the algorithm proposed in this paper.

Figure 8 shows a comparison of computing accuracy for different interpolation function approximation. The boundary shape is represented by Lagrange linear, quadratic shape function and NURBS curve of 2 degree, respectively. The approximation of physical quantities is implemented by piecewise constant discretization with linear shape approximation (called Lagrange-DBE21), piecewise linear discretization with linear shape approximation (called Lagrange-DBE22), piecewise quadratic discretization with quadratic shape approximation (called Lagrange-DBE33) and NURBS basis function discretization (called NURBS-D2), respectively. Herein, the relative error denotes the surface error based on Euclidean norm, see Ref. 43 for detailed content. A circle with a radius $r=2 \mathrm{~m}$ is built. Around 360 computing points distributed on the circle uniformly are used to discretize the circle and compute the surface error. By observing this figure, we can find that: the relative error decreases with increasing DOFs for all different types of the BEM; higher order Lagrange function interpolation produces numerical solution with higher accuracy than lower Lagrange function interpolation; NURBS basis function interpolation performs better than Lagrange function interpolation with same order and DOFs by comparing Lagrange-DBE33 with NURBS-D2. Figure 9 shows a comparison of computing accuracy 
L. Chen et al.

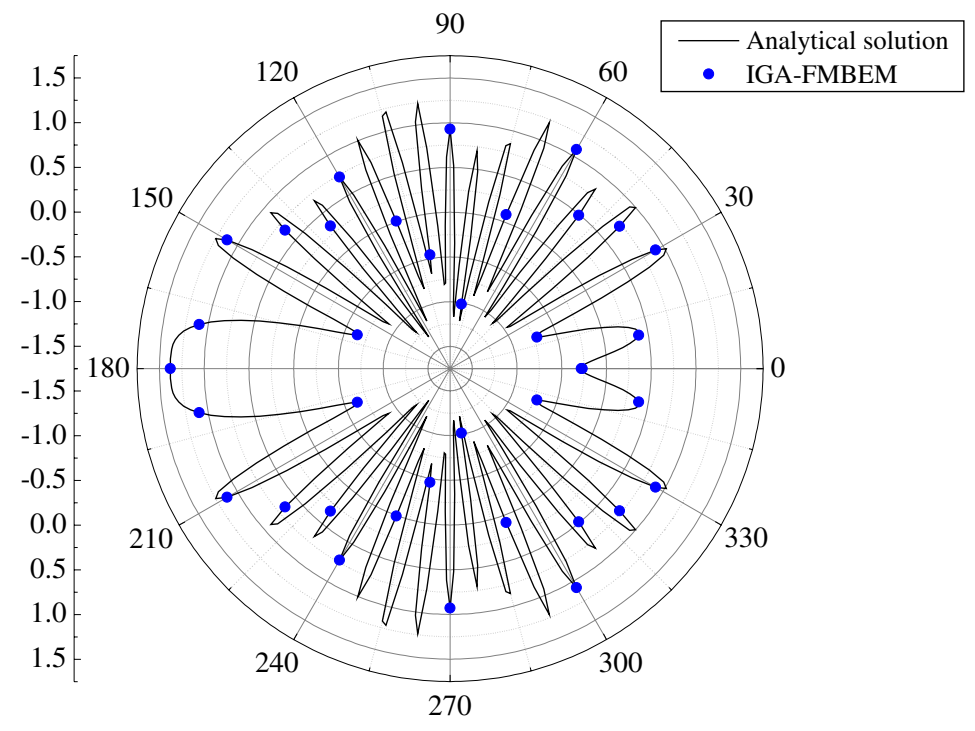

(a) Real part

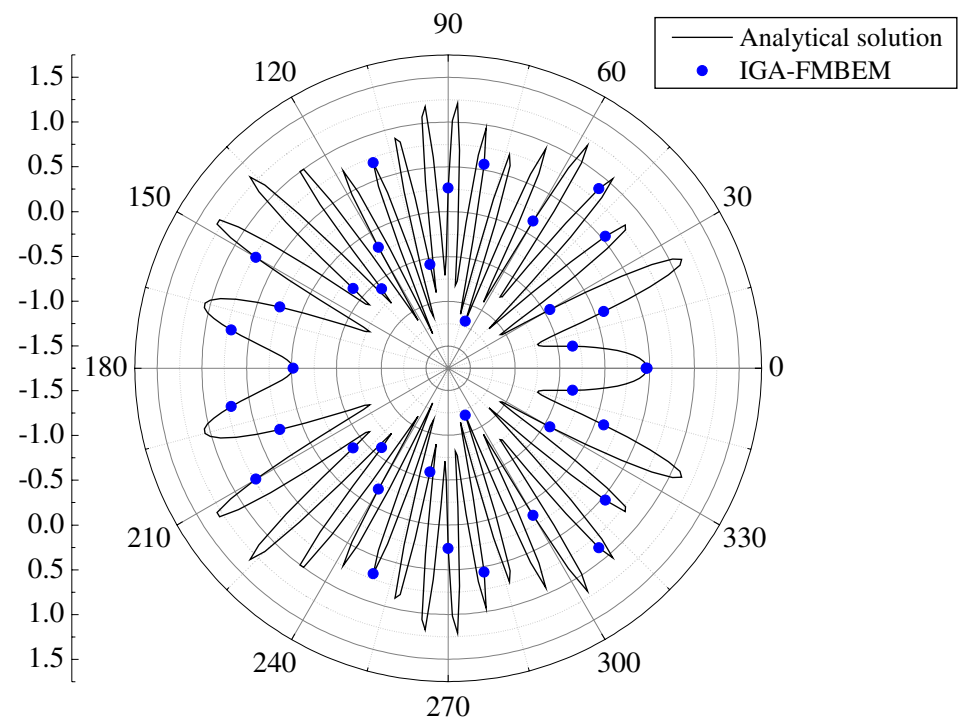

(b) Imaginary part

Fig. 5. Sound pressure at test points distributed on a circle with a radius $r=4$, at $k a=4 \pi$.

for different types of BEM with frequency, where the DOFs is set as 5000. The symbol "CBIE" denotes the single boundary integral equation which is used for the solution of the problem, and "BM" denotes the Burton-Miller method which is applied to solve the problem. The result that NURBS interpolation element performs better than Lagrange interpolation element can also be found in Fig. 9. On the other hand, we can find that 


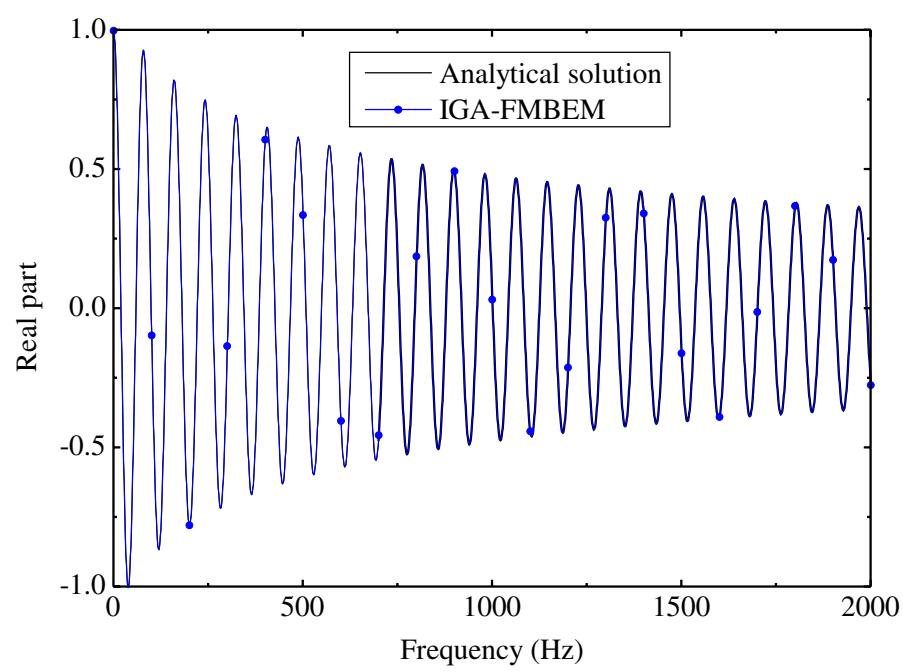

Fig. 6. Real part of sound pressure at a test point located on $(4,0)$ with frequency.

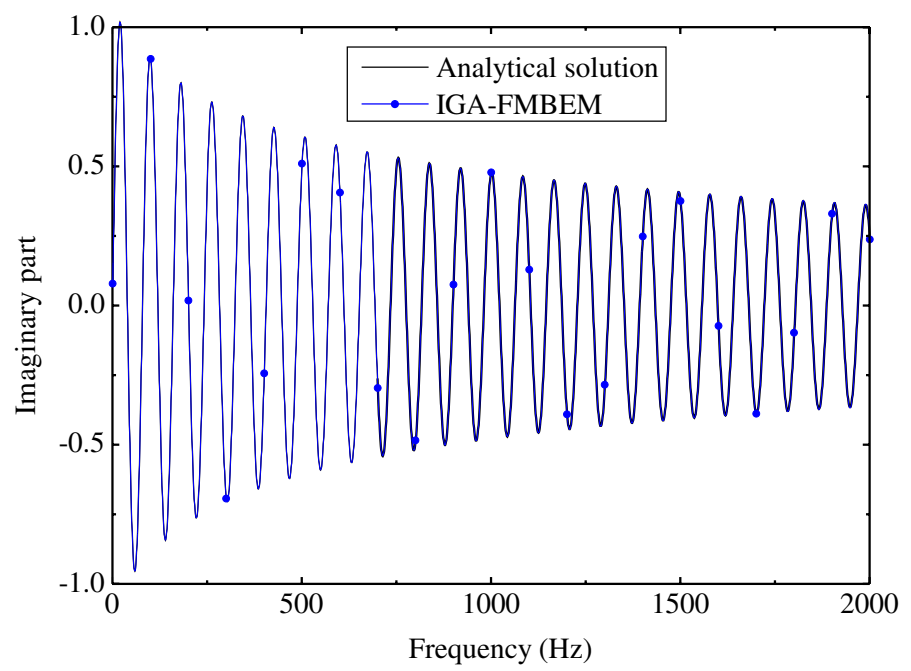

Fig. 7. Imaginary part of sound pressure at a test point located on $(4,0)$ with frequency.

the peaks originate from the spurious eigen-frequencies. But, Burton-Miller method can be used successfully to overcome this problem by observing the results of NURBS-D2 (CBIE) and NURBS-D2 (BM), where the couple factor is chosen as $i / k$ for $k \geq 1$, but $i$ for $k<1$.

A key point of this paper consists in applying the FMM into the IGA-BEM for 2D acoustic problem to accelerate the solution of the IGA-BEM. Figure[10]shows that the use of the FMM improves efficiently the computing performance of IGA-BEM, and it demonstrates the high efficiency of the algorithm proposed in this paper. On the other hand, we can 
L. Chen et al.

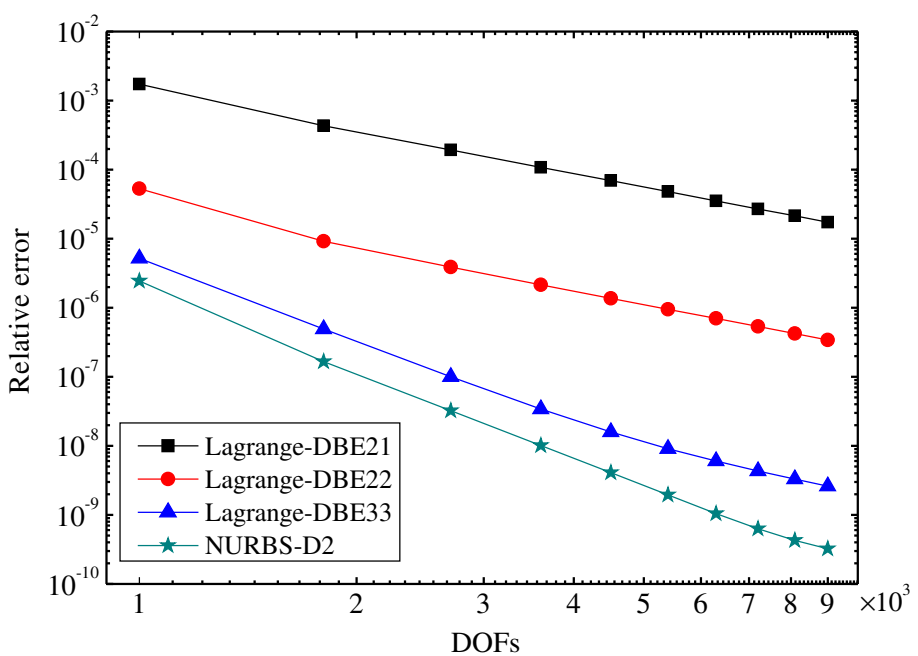

Fig. 8. Comparison of computing accuracy for different types of BEM with DOFs: Lagrange functions and NURBS basis function are used for the boundary shape approximation and physics field approximation, respectively.

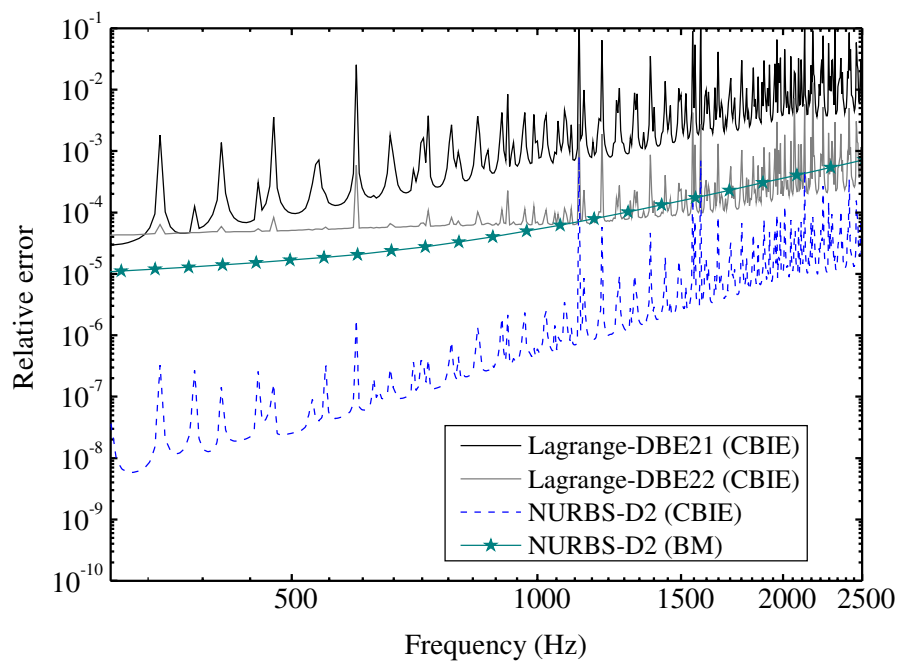

Fig. 9. Comparison of computing accuracy for different types of BEM with frequency: the DOF is 5000.

find that the use of IGA consumes more computing time by comparing the results of the IGA-FMBEM and the FMBEM. That is because the derived formulas for the NURBS basis functions are recursive and takes much more computing time. Actually, in their current form, the expressions of NURBS basis functions are more expensive than that of the conventional polynomial basis function. The direct solution will reduce the computational efficiency. However, there exist several efficient algorithms for their evaluation, such as the extraction 


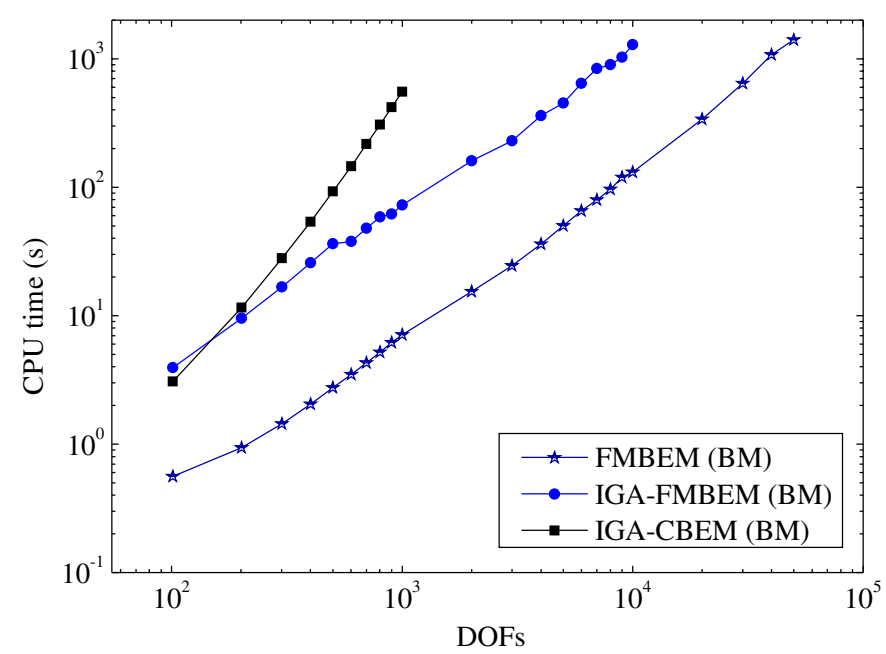

Fig. 10. Comparison of CPU time used to calculate the sound pressure at test point $(4,0)$ with frequency $f=2000 \mathrm{~Hz}$.

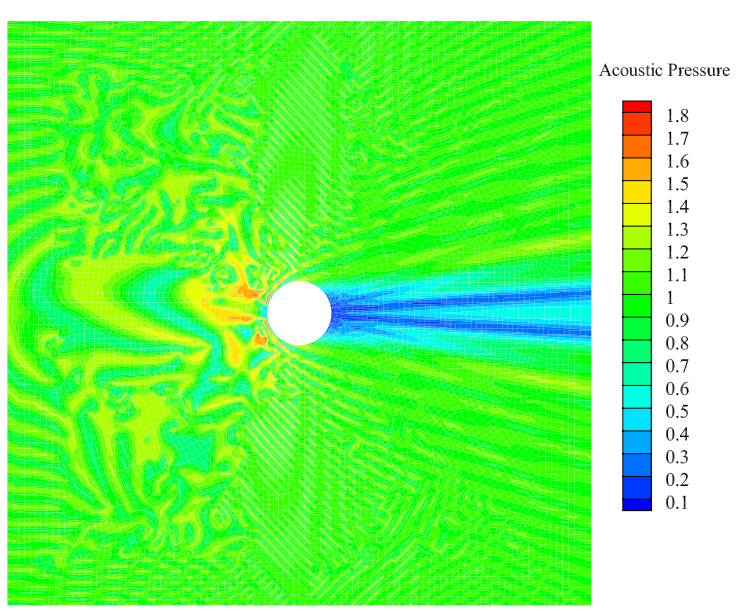

(a) Single scatter

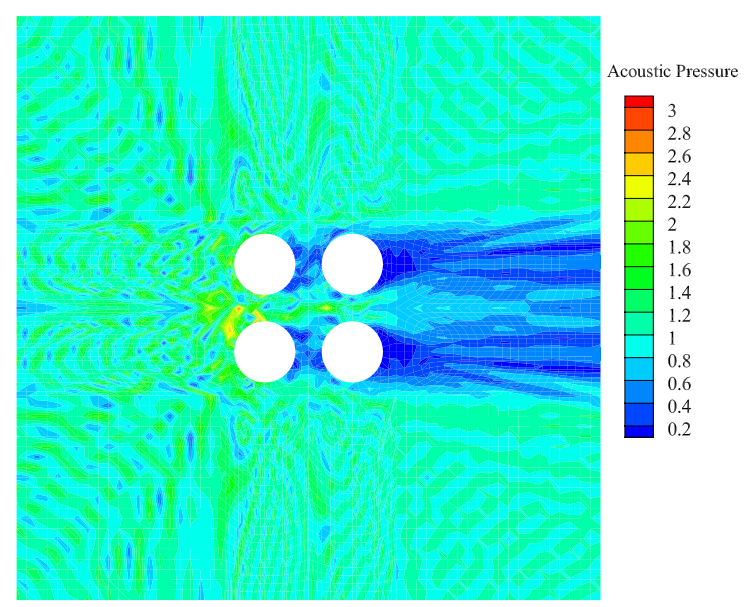

(b) Four scatters

Fig. 11. Sound pressure contour plot for scattering of single and four cylinders with $f=1000 \mathrm{~Hz}$.

operator,$\underline{48}$ and the Cox-de-Boor algorithm ${ }^{49}$ In spite of this, we still develop the applicable of IGA because of its high accuracy and flexibility for shape change.

Figure 11] shows the sound pressure contour for scattering field for single and four cylindrical shells problems with $f=1000 \mathrm{~Hz}$, respectively. Figure 12 shows the sound pressure contour for scattering field for octagonal column and eight blade column with $f=1000 \mathrm{~Hz}$, respectively. These figures show the high applicability of the algorithm proposed in this paper for complex problems. 


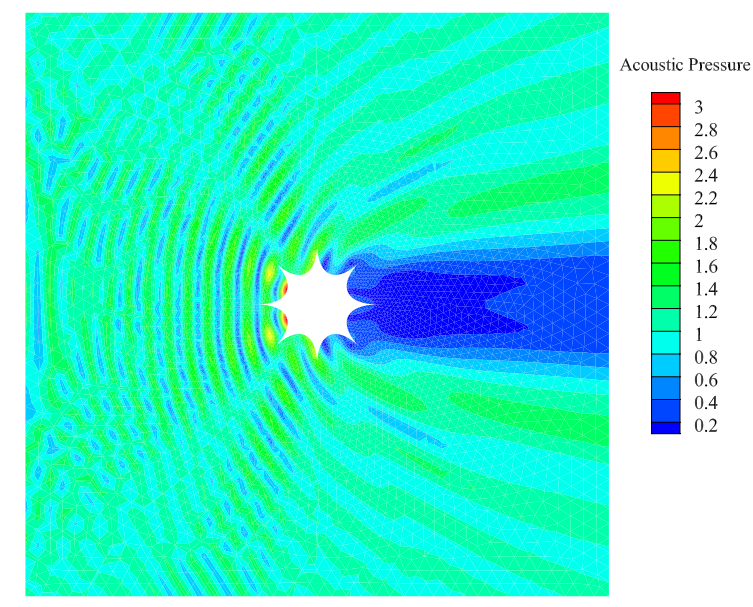

(a) Octagonal column

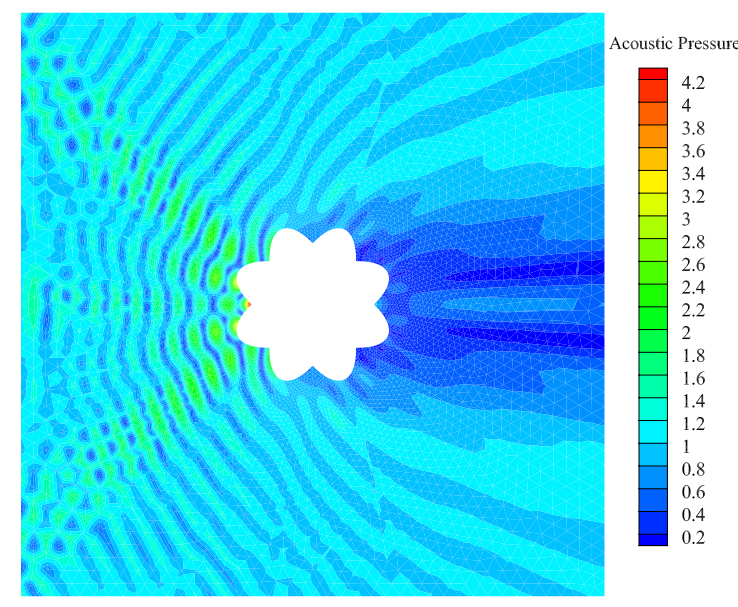

(b) Eight blade column

Fig. 12. Sound pressure contour plot for scattering of octagonal column and eight blade column with $f=$ $1000 \mathrm{~Hz}$.

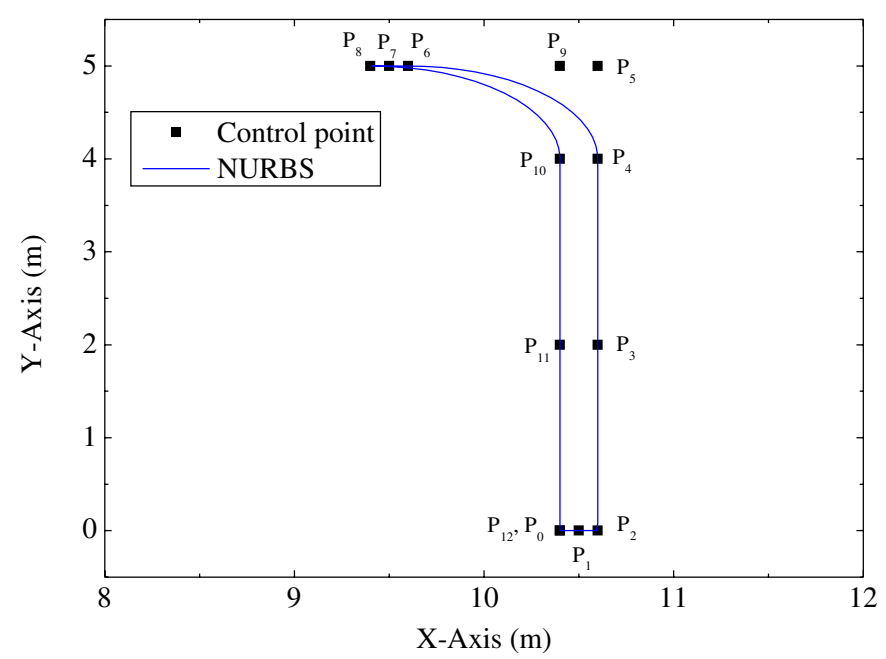

Fig. 13. Cross-section of the noise barrier with curved top structure represented by NURBS of 2 degree with 13 control points: the knot vector $\Xi=\{0,0,0,1 / 6,1 / 6,2 / 6,2 / 6,3 / 6,3 / 6,4 / 6,4 / 6,5 / 6,5 / 6,1,1,1\}$.

In order to demonstrate the high validity and efficiency of the algorithm proposed in this paper for 2D half-space acoustic problems with absorbing material, an example of noise barrier with curved top structure is tested, see Fig. 13. The data about the coordinate of control points and the associated weight is listed in Table 2. Absorbing material is located on the noise barrier, and the associated normalized surface admittance is set as 1. Figure 14 shows the SPL contour plot for scattering of noise barrier with curved top structure with frequency $f=100 \mathrm{~Hz}$. 
Table 2. Data about control point and the associated weight for noise barrier with curved top structure.

\begin{tabular}{cccc}
\hline Control Point & Coordinate in $\mathbf{X}$-Axis & Coordinate in $\mathbf{Y}$-Axis & Weight $w$ \\
\hline $\mathrm{P}_{0}$ & 0.0 & 0.0 & 1.0 \\
$\mathrm{P}_{1}$ & 0.1 & 0.0 & 1.0 \\
$\mathrm{P}_{2}$ & 0.2 & 0.0 & 1.0 \\
$\mathrm{P}_{3}$ & 0.2 & 2.0 & 1.0 \\
$\mathrm{P}_{4}$ & 0.2 & 4.0 & 1.0 \\
$\mathrm{P}_{5}$ & 0.2 & 5.0 & $1 / \sqrt{2}$ \\
$\mathrm{P}_{6}$ & -0.8 & 5.0 & 1.0 \\
$\mathrm{P}_{7}$ & -0.9 & 5.0 & 1.0 \\
$\mathrm{P}_{8}$ & -1.0 & 5.0 & 1.0 \\
$\mathrm{P}_{9}$ & 0.0 & 5.0 & $1 / \sqrt{2}$ \\
$\mathrm{P}_{10}$ & 0.0 & 4.0 & 1.0 \\
$\mathrm{P}_{11}$ & 0.0 & 2.0 & 1.0 \\
$\mathrm{P}_{12}$ & 0.0 & 0.0 & 1.0 \\
\hline
\end{tabular}

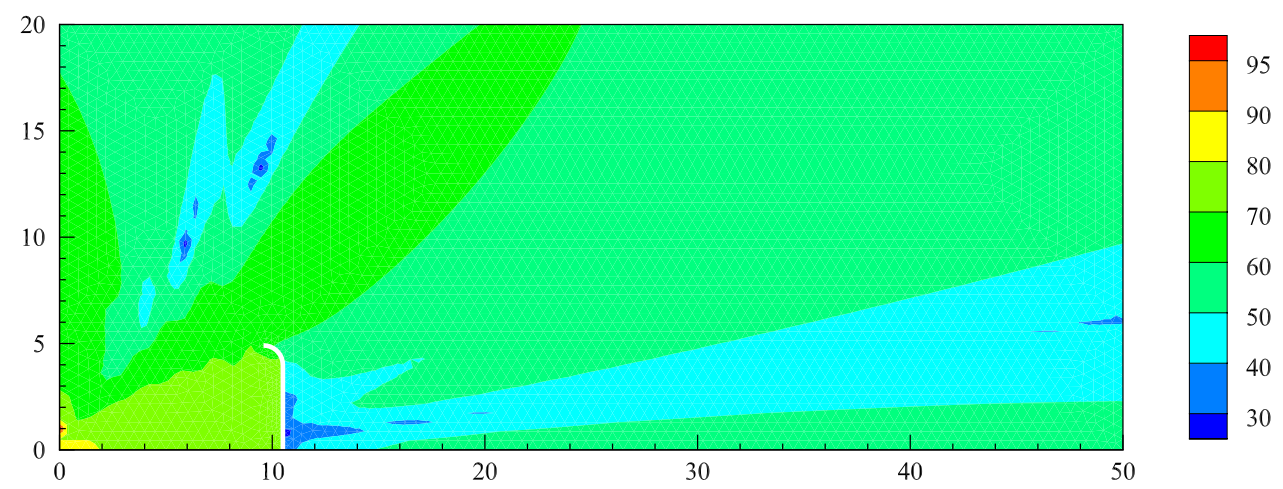

Fig. 14. SPL contour plot for scattering of noise barrier with curved top structure based on IGA-FMBEM.

\section{Conclusion}

A novel algorithm based on the IGA-FMBEM is presented for the simulation of 2D halfspace acoustic scattering problems with admittance boundary conditions. The Burton and Miller method is used to get correct solutions at all frequencies. A cylinder example for which an analytical solution is available is chosen to demonstrate the correctness and validity of the proposed algorithm, and the performance of different types of boundary elements is presented. The result shows that the proposed method compares very favorably, exhibiting a significantly higher accuracy than the conventional Lagrangian BEM with same degree of freedom.

Future work will further extend the proposed algorithm into 2D acoustic optimization analysis and 3D half-space acoustic problems. For 3D acoustic problems, one of the greatest difficulties is to eliminate the singularity of the boundary integrals. we will focus on overcoming this problem. 


\section{Chen et al.}

\section{Acknowledgments}

This study was funded by the National Natural Science Foundation of China (NSFC) under Grant No. 11702238, Henan Provincial Department of Science and Technology Research under Grant no. 172102210453, Key Scientific Research Project of Henan University under Grant no. 17A560009, and Nanhu Scholars Program for Young Scholars of XYNU.

\section{References}

1. S. Marburg, Developments in structural-acoustic optimization for passive noise control, Arch. Comput. Methods Eng. 27 (2002) 291-370.

2. C. A. Brebbia, J. Dominguez and J. L. Tassoulas, Boundary elements: An introductory course, Comput. Mech. Publ. 58 (1989) 860.

3. Y. J. Liu, S. Mukherjee, N. Nishimura, M. Schanz, W. Ye, A. Sutradhar, E. Pan, N. A. Dumont, A. Frangi and A. Saez, Recent advances and emerging applications of the boundary element method, Appl. Mech. Rev. 64 (2011) 031001.

4. N. A. Gumerov and R. Duraiswami, Fast multipole methods on graphics processors, J. Comput. Phys. 227 (2008) 8290-8313.

5. V. Rokhlin, Rapid solution of integral equations of scattering theory in two dimensions, $J$. Comput. Phys. 86 (1990) 414-439.

6. L. Greengard and V. Rokhlin, A fast algorithm for particle simulations, J. Comput. Phys. 73 (1987) 325-348.

7. L. L. Chen, C. J. Zheng and H. B. Chen, A wideband FMBEM for 2D acoustic design sensitivity analysis based on direct differentiation method, Comput. Mech. 52 (2013) 631-648.

8. L. L. Chen, C. J. Zheng and H. B. Chen, FEM/wideband FMBEM coupling for structuralacoustic design sensitivity analysis, Comput. Methods Appl. Mech. Eng. 276 (2014) 1-19.

9. C. J. Zheng, T. Matsumoto, T. Takahashi and H. B. Chen, A wideband fast multipole boundary element method for three dimensional acoustic shape sensitivity analysis based on direct differentiation method, Eng. Anal. Bound. Elem. 36 (2012) 361-371.

10. S. Marburg, Six boundary elements per wavelength. Is that enough? J. Comput. Acoust. 10 (2002) 25-51.

11. L. L. Chen, S. Marburg, H. B. Chen, H. Zhang and H. B. Gao, An adjoint operator approach for sensitivity analysis of radiated sound power in fully coupled structural-acoustic systems, $J$. Comput. Acoust. 25 (2017) 1750003.

12. L. L. Chen, H. B. Chen, C. J. Zheng and S. Marburg, Structuralacoustic sensitivity analysis of radiated sound power using a finite element/discontinuous fast multipole boundary element scheme, Int. J. Numer. Methods Fluids 82 (2016) 858-878.

13. A. J. Burton and G. F. Miller, The application of integral equation methods to the numerical solution of some exterior boundary-value problem, Proc. R. Soc. Lond. A 323 (1971) 201210.

14. C. J. Zheng, H. B. Chen, H. F. Gao and L. Du, Is the Burton-Miller formulation really free of fictitious eigenfrequencies, Eng. Anal. Bound. Elem. 59 (2015) 43-51.

15. S. Marburg, The Burton and Miller method: Unlocking another mystery of its coupling parameter, J. Comput. Acoust. 24 (2016) 1550016.

16. T. J. R. Hughes, J. A. Cottrell and Y. Bazilevs, Isogeometric analysis: CAD, finite elements, NURBS, exact geometry and mesh refinement, Comput. Methods Appl. Mech. Eng. 194 (2005) 4135-4195. 
17. V. P. Nguyen, C. Anitescu, S. P. A. Bordas and T. Rabczuk, Isogeometric analysis: An overview and computer implementation aspects, Math. Comput. Simul. 117 (2015) 89-116.

18. J. A. Cottrell, T. J. R. Hughes and Y. Bazilevs, Isogeometric Analysis: Toward Integration of CAD and FEA (Wiley Publishing, 2009), pp. 299-325.

19. K. A. Johannessen, T. Kvamsdal and T. Dokken, Isogeometric analysis using LR B-splines, Comput. Methods Appl. Mech. Eng. 269 (2014) 471-514.

20. T. Takahashi and T. Matsumoto, An application of fast multipole method to isogeometric boundary element method for Laplace equation in two dimensions, Eng. Anal. Bound. Elem. 36 (2012) 1766-1775.

21. Y. P. Gong, C. Y. Dong and X. C. Qin, An isogeometric boundary element method for three dimensional potential problems, J. Comput. Appl. Math. 313 (2017) 454-468.

22. J. L. Gu, J. M. Zhang and G. Y. Li, Isogeometric analysis in BIE for 3-D potential problem, Eng. Anal. Bound. Elem. 36 (2012) 858-865.

23. C. Politis, A. I. Ginnis, P. D. Kaklis and K. Belibassakis, C. Feurer, An isogeometric BEM for exterior potential-flow problems in the plane, in SIAM/ACM Joint Conf. Geometric and Physical Modeling (ACM, NY, 2009), pp. 349-354.

24. R. N. Simpson, S. P. A. Bordas and J. Trevelyan, T. Rabczuk, A two-dimensional Isogeometric Boundary Element Method for elastostatic analysis, Comput. Methods Appl. Mech. Eng. 209 (2012) 87-100.

25. R. N. Simpson, S. P. A. Bordas, L. He and J. Trevelyan, An isogeometric boundary element method for elastostatic analysis: 2D implementation aspects, Comput. Struct. 118 (2013) $2-12$.

26. B. H. Nguyen, H. D. Tran, C. Anitescu, X. Zhuang and T. Rabczuk, An isogeometric symmetric Galerkin boundary element method for two-dimensional crack problems, Comput. Methods Appl. Mech. Eng. 306 (2016) 252-275.

27. X. Peng, E. Atroshchenko, P. Kerfriden and S. P. A. Bordas, Isogeometric boundary element methods for three dimensional static fracture and fatigue crack growth, Comput. Methods Appl. Mech. Eng. 316 (2016) 151-185.

28. M. Feischl, T. Fhrer, N. Heuer, M. Karkulik and D. Praetorius, Adaptive boundary element methods, Arch. Comput. Methods Eng. 22 (2015) 309-389.

29. M. J. Peake, J. Trevelyan and G. Coates, Extended isogeometric boundary element method (XIBEM) for two-dimensional Helmholtz problems, Comput. Methods Appl. Mech. Eng. 259 (2013) 93-102.

30. M. J. Peake, J. Trevelyan and G. Coates, Extended isogeometric boundary element method (XIBEM) for three-dimensional medium-wave acoustic scattering problems, Comput. Methods Appl. Mech. Eng. 284 (2015) 762-780.

31. R. N. Simpson, M. A. Scott, M. Taus, D. C. Thomas and H. Lian, Acoustic isogeometric boundary element analysis, Comput. Methods Appl. Mech. Eng. 269 (2014) 265-290.

32. L. Coox, O. Atak, D. Vandepitte and W. Desmet, An isogeometric indirect boundary element method for solving acoustic problems in open-boundary domains, Comput. Methods Appl. Mech. Eng. 316 (2017) 186-208.

33. K. A. Belibassakis, Th. P. Gerostathis, K. V. Kostas, C. G. Politis, P. D. Kaklis, A. I. Ginnis and C. Feurer, A BEM-isogeometric method for the ship wave-resistance problem, Ocean Eng. 60 (2013) 53-67.

34. A. I. Ginnis, K. V. Kostas, C. G. Politis, P. D. Kaklis, K. A. Belibassakis, Th. P. Gerostathis, M. A. Scott and T. J. R. Hughes, Isogeometric boundary-element analysis for the wave-resistance problem using T-splines, Comput. Methods Appl. Mech. Eng. 279 (2014) 425-439. 
L. Chen et al.

35. K. V. Kostas, A. I. Ginnis, C. G. Politis and P. D. Kaklis, Ship-hull shape optimization with a T-spline based BEM-isogeometric solver, Comput. Methods Appl. Mech. Eng. 284 (2015) 611-622.

36. H. Lian, P. Kerfriden and S. P. A. Bordas, Shape optimization directly from CAD: An isogeometric boundary element approach using T-splines, Comput. Methods Appl. Mech. Eng. 317 (2017) 1-41.

37. S. W. Lee, M. Yoon and S. Cho, Isogeometric topological shape optimization using dual evolution with boundary integral equation and level sets, Computer-Aided Design. 82 (2017) 88-99.

38. W. A. Wall, M. A. Frenzel and C. Cyron, Isogeometric structural shape optimization, Comput. Methods Appl. Mech. Eng. 197 (2008) 2976-2988.

39. S. Cho and S. H. Ha, Isogeometric shape design optimization: Exact geometry and enhanced sensitivity, Struct. Multidisc. Optim. 38 (2009) 53-70.

40. A. P. Nagy, M. M. Abdalla and Z. Gürdal, Isogeometric sizing and shape optimization of beam structures, Comput. Methods Appl. Mech. Eng. 199 (2010) 1216-1230.

41. N. D. Manh, A. Evgrafov, A. R. Gersborg and J. Gravesen, Isogeometric shape optimization of vibrating membranes, Comput. Methods Appl. Mech. Eng. 200 (2011) 1343-1353.

42. J. Kiendl, R. Schmidt, R. Wüchner and K. U. Bletzinger, Isogeometric shape optimization of shells using semi-analytical sensitivity analysis and sensitivity weighting, Comput. Methods Appl. Mech. Eng. 274 (2014) 148-167.

43. S. Marburg and S. Schneider, Influence of element types on numeric error for acoustic boundary elements, J. Comput. Acoust. 11 (2003) 363-386.

44. L. L. Chen, L. C. Liu, W. C. Zhao and H. B. Chen, 2D acoustic design sensitivity analysis based on adjoint variable method using different types of boundary elements, Australia Acoust. 44 (2016) 343-357.

45. M. S. Bapat, L. Shen and Y. J. Liu, Adaptive fast multipole boundary element method for three-dimensional half-space acoustic wave problems, Eng. Anal. Bound. Elem. 33 (2009) 11131123.

46. D. Brunner, Fast boundary element methods for large-scale simulations of the vibro-acoustic behavior of ship-like structures, PhD thesis, University of Stuttgart (2009).

47. Y. Yasuda, K. Higuchi, T. Oshima and T. Sakuma, Efficient technique in low-frequency fast multipole boundary element method for plane-symmetric acoustic problems, Eng. Anal. Bound. Elem. 36 (2012) 1493-1501.

48. M. A. Scott, M. J. Borden, C. V. Verhoosel, T. W. Sederberg and T. J. R. Hughes, Isogeometric finite element data structures based on Bézier extraction of t-splines, Int. J. Numer. Methods Eng. 88 (2011) 126-156.

49. V. Popov and H. Power, An $\mathrm{O}(\mathrm{N})$ taylor series multipole boundary element method for threedimensional elasticity problems, Eng. Anal. Bound. Elem. 25 (2001) 7-18.

\section{Appendix A: Evaluation of Singular Boundary Integrals}

The kernel functions in Eqs. (29) and (30) are expressed as

$$
\begin{gathered}
G=\frac{i}{4} H_{0}^{(1)}(k \bar{r})+\frac{i}{4} H_{0}^{(1)}(k \hat{r})=G_{1}+\bar{G}_{1} \\
\frac{\partial G}{\partial n\left(r_{s}\right)}=-\frac{i k}{4} H_{1}^{(1)}(k \bar{r}) \frac{\partial \bar{r}}{\partial n\left(r_{s}\right)}-\frac{i k}{4} H_{1}^{(1)}(k \hat{r}) \frac{\partial \hat{r}}{\partial n\left(r_{s}\right)},
\end{gathered}
$$




$$
\begin{aligned}
\frac{\partial G}{\partial n(r)} & =-\frac{i k}{4} H_{1}^{(1)}(k \bar{r}) \frac{\partial \bar{r}}{\partial n(r)}-\frac{i k}{4} H_{1}^{(1)}(k \hat{r}) \frac{\partial \hat{r}}{\partial n(r)}, \\
\frac{\partial^{2} G}{\partial n(r) \partial n\left(r_{s}\right)} & =G_{2}+\bar{G}_{2},
\end{aligned}
$$

where $H_{n}^{(1)}$ denotes the first hankel function of the $n$th order, $\bar{r}=\left|r-r_{s}\right|$, and $\hat{r}$ denotes the distance between the mirror point of source point $r$ and field point $r_{s}$. $G_{2}$ and $\bar{G}_{2}$ can be expressed as

$$
G_{2}=\frac{i k}{4 \bar{r}} H_{1}^{(1)}(k \bar{r}) n_{l}(r) n_{l}\left(r_{s}\right)+\frac{i k^{2}}{4} H_{2}^{(1)}(k \bar{r}) \frac{\partial \bar{r}}{\partial n(r)} \frac{\partial \bar{r}}{\partial n\left(r_{s}\right)}
$$

and

$$
\bar{G}_{2}=\frac{i k}{4 \hat{r}} H_{1}^{(1)}(k \hat{r}) n_{l}(r) n_{l}\left(r_{s}\right)+\frac{i k^{2}}{4} H_{2}^{(1)}(k \hat{r}) \frac{\partial \hat{r}}{\partial n(r)} \frac{\partial \hat{r}}{\partial n\left(r_{s}\right)}
$$

when $r \rightarrow 0$, the singularity order of the kernel functions in Eqs. (29) and (30) are given as follows

$$
H_{0}^{(1)}(\bar{r}) \sim \frac{2 i}{\pi} \ln (\bar{r}), \quad H_{1}^{(1)}(\bar{r}) \sim-\frac{2 i}{\pi \bar{r}}, \quad H_{2}^{(1)}(\bar{r}) \sim-\frac{4 i}{\pi \bar{r}^{2}} .
$$

Due to the same order of $\frac{\partial \bar{r}}{\partial n(r)}, \frac{\partial \bar{r}}{\partial n\left(r_{s}\right)}$ and $\bar{r}$, only the singular integrals for the kernel functions $G$ and $\frac{\partial^{2} G}{\partial n(r) \partial n\left(r_{s}\right)}$ need to be evaluated. Herein, the Cauchy principal value and the Hadamard finite part integral method are used to evaluate the singular integrals. Firstly, the nonsingular integral expression for the kernel function $G_{1}$ is derived by

$$
\begin{aligned}
\int_{\xi_{e}}^{\xi_{e+1}} & G_{1} R_{j, p_{f}}(\xi) J(\xi) d \xi \\
= & \frac{i}{4} \int_{\xi_{e}}^{\xi_{e+1}}\left[H_{0}^{(1)}(k \bar{r}) R_{j, p_{f}}(\xi) J(\xi) \frac{2 i}{\pi} \ln (k|\xi-\bar{\xi}| J(\bar{\xi})) R_{j, p_{f}}(\bar{\xi}) J(\bar{\xi})\right] d \xi \\
& -\frac{1}{2 \pi} \int_{\xi_{e}}^{\xi_{e+1}} \ln (k|\xi-\bar{\xi}| J(\bar{\xi})) R_{j, p_{f}}(\bar{\xi}) J(\bar{\xi}) d \xi
\end{aligned}
$$

where the first term in the right-hand side of the above equation is nonsingular and can be solved by Gauss-Legendre integral method. The second term is weakly singular and can be evaluated directly, as follows:

$$
\begin{aligned}
& \frac{1}{2 \pi} \int_{\xi_{e}}^{\xi_{e+1}} \ln (k|\xi-\bar{\xi}| J(\bar{\xi})) R_{j, p_{f}}(\bar{\xi}) J(\bar{\xi}) d \xi \\
& \quad=\frac{1}{2 \pi} R_{j, p_{f}}(\bar{\xi}) J(\bar{\xi})\left(\xi_{e+1}-\xi_{e}\right) \ln (k J(\bar{\xi}))+\frac{1}{2 \pi} R_{j, p_{f}}(\bar{\xi}) J(\bar{\xi})\left[\left(\bar{\xi}-\xi_{e}\right) \ln \left(\bar{\xi}-\xi_{e}\right)\right. \\
& \left.\quad+\left(\xi_{e+1}-\bar{\xi}\right) \ln \left(\xi_{e+1}-\bar{\xi}\right)-\left(\xi_{e+1}-\xi_{e}\right)\right]
\end{aligned}
$$




\section{Chen et al.}

Replacing the source point $r$ with its mirror point in Eqs. (A.8) and (A.9), we can obtain the nonsingular integral expression for the kernel function $\bar{G}_{1}$.

Similarly, the nonsingular integral expression for the kernel function $G_{2}$ is derived by

$$
\begin{aligned}
\frac{i k}{4} \int_{\xi_{e}}^{\xi_{e+1}} & \frac{H_{1}^{(1)}(k \bar{r})}{\bar{r}} n_{l}(r) n_{l}\left(r_{s}\right) R_{j, p_{f}}(\xi) J(\xi) d \xi \\
= & \frac{i k^{2}}{4} \int_{\xi_{e}}^{\xi_{e+1}}\left[\frac{H_{1}^{(1)}(k \bar{r})}{k \bar{r}} n_{l}(r) n_{l}\left(r_{s}\right) R_{j, p_{f}}(\xi) J(\xi)\right. \\
& \left.+\left(\frac{2 i}{\pi k^{2}(|\xi-\bar{\xi}| J(\bar{\xi}))^{2}}-\frac{i}{\pi} \ln (k|\xi-\bar{\xi}| J(\bar{\xi}))\right) R_{j, p_{f}}(\bar{\xi}) J(\bar{\xi})\right] d \xi \\
& -\frac{i k^{2}}{4} \int_{\xi_{e}}^{\xi_{e+1}}\left[\frac{2 i}{\pi k^{2}(|\xi-\bar{\xi}| J(\bar{\xi}))^{2}}-\frac{i}{\pi} \ln (k|\xi-\bar{\xi}| J(\bar{\xi}))\right] R_{j, p_{f}}(\bar{\xi}) J(\bar{\xi}) d \xi
\end{aligned}
$$

where the first term in the right-hand side of the above equation is nonsingular and can be solved by Gauss-Legendre integral method. The second term is singular and can be evaluated directly, as follows:

$$
\begin{aligned}
& \frac{i k^{2}}{4} \int_{\xi_{e}}^{\xi_{e+1}}\left[\frac{2 i}{\pi k^{2}(|\xi-\bar{\xi}| J(\bar{\xi}))^{2}}-\frac{i}{\pi} \ln (k|\xi-\bar{\xi}| J(\bar{\xi}))\right] R_{j, p_{f}}(\bar{\xi}) J(\bar{\xi}) d \xi \\
& =-\frac{R_{j, p_{f}}(\bar{\xi})}{2 \pi J(\bar{\xi})}\left(\frac{1}{\xi_{e}-\bar{\xi}}-\frac{1}{\xi_{e+1}-\bar{\xi}}\right)+\frac{k^{2}}{4 \pi} R_{j, p_{f}}(\bar{\xi}) J(\bar{\xi})\left(\xi_{e+1}-\xi_{e}\right) \ln (k J(\bar{\xi})) \\
& \quad+\frac{k^{2}}{4 \pi} R_{j, p_{f}}(\bar{\xi}) J(\bar{\xi})\left[\left(\bar{\xi}-\xi_{e}\right) \ln \left(\bar{\xi}-\xi_{e}\right)+\left(\xi_{e+1}-\bar{\xi}\right) \ln \left(\xi_{e+1}-\bar{\xi}\right)-\left(\xi_{e+1}-\xi_{e}\right)\right]
\end{aligned}
$$

Replacing the source point $r$ with its mirror point in Eq. A.10), we can obtain the nonsingular integral expression for the kernel function $\bar{G}_{2}$. 